Article

\title{
Energy Savings Results from Small Commercial Building Retrofits in the US
}

\author{
Rachael Sherman ${ }^{1}$, Hariharan Naganathan ${ }^{2}$ and Kristen Parrish ${ }^{3, *}$ \\ 1 Engineering Technology and Construction Management, University of North Carolina, \\ Charlotte, NC 28223, USA; rsherma6@uncc.edu \\ 2 Construction Management, School of Management, Wentworth Institute of Technology, \\ Boston, MA 02115, USA; naganathanh@wit.edu \\ 3 School of Sustainable Engineering and the Built Environment, Arizona State University, Tempe, AZ 85287, USA \\ * Correspondence: Kristen.Parrish@asu.edu; Tel.: +1-480-727-6363
}

Citation: Sherman, R.; Naganathan, H.; Parrish, K. Energy Savings Results from Small Commercial Building Retrofits in the US. Energies 2021, 14 , 6207. https://doi.org/10.3390/ en14196207

Academic Editor: Dimitrios Katsaprakakis

Received: 31 May 2021

Accepted: 14 September 2021

Published: 29 September 2021

Publisher's Note: MDPI stays neutral with regard to jurisdictional claims in published maps and institutional affiliations.

Copyright: (c) 2021 by the authors. Licensee MDPI, Basel, Switzerland. This article is an open access article distributed under the terms and conditions of the Creative Commons Attribution (CC BY) license (https:// creativecommons.org/licenses/by/ $4.0 /)$.

\begin{abstract}
Small commercial buildings, or those comprising less than 50,000 square feet of floor area, represent $94 \%$ of U.S commercial buildings by count and consume approximately $8 \%$ of the nation's primary energy; as such, they represent a largely unexploited opportunity for energy savings. Small commercial buildings also represent a large economic market-the National Institute of Building Sciences (NIBS) estimated the small commercial retrofit market at USD 35.6 billion. Despite the prominence of small commercial buildings and the economic opportunity for energy retrofits, many energy efficiency programs focus on large commercial buildings, and create efficiency solutions that do not meet the needs of the small commercial market. This paper presents an analysis of 34 small commercial case study projects that implemented energy efficiency retrofits. This paper contributes to the existing building retrofit body of knowledge in the following ways: (1) it identifies the decision criteria used by small commercial building stakeholders that decided to complete an energy retrofit; (2) it identifies the most commonly implemented efficiency measures in small commercial buildings, and discusses why this is the case; and (3) it provides empirical evidence about the efficacy of installing single energy efficiency measures (EEMs) compared to packages of EEMs in small commercial buildings by reporting verified energy savings. To the authors' knowledge, this paper is the first to catalog decision criteria and energy savings for the existing small commercial buildings market, and this research illustrates that small commercial building decision-makers seem most motivated to retrofit their spaces by energy cost savings and operational concerns. Furthermore, small commercial building decision-makers opted to implement single-system retrofits in fifteen (15) of the thirty-four cases studied. Finally, this research documents the improved savings, in the small commercial buildings market, associated with a more integrated package of EEMs compared to a single-system approach, achieving approximately $10 \%$ energy savings for a single-system approach and more than $20 \%$ energy savings for integrated approaches. These savings translate to $\mathrm{CO}_{2}$ savings of $1,324,000 \mathrm{kgCO}_{2}$ / year to $2,647,000 \mathrm{kgCO}_{2}$ /year, respectively, assuming small commercial buildings are retrofit at a rate of $0.95 \%$ of the stock annually.
\end{abstract}

Keywords: small commercial; retrofit; energy efficiency

\section{Introduction}

Small commercial buildings, or those that comprise less than $50,000 \mathrm{ft}^{2}$ of usable floor space, make up approximately $94 \%$ of the US building stock and consume about $8 \%$ of the US' primary energy [1] and as previous research has identified, profitable investments in energy conservation can generate more than USD 30 billion in annual energy cost savings, and, as such, improving the financial performance of many small businesses throughout the United States [2-4]. However, there is a deficiency of resources available to small commercial building owners who are interested in retrofitting their buildings. Although sustainability and energy conservation are often staples in large commercial building 
owners' portfolios, it has yet to be identified what drives small commercial building owners to complete energy efficiency retrofits in their buildings or portfolios. To address this deficiency, the authors present the decision criteria, retrofit measures implemented, and the energy savings results of the retrofits of 34 small commercial buildings, as reported in case studies.

\subsection{The Case for Energy Efficiency Retrofits}

With the commercial building sector consuming nearly 30\% of all US energy [5], the "Better Building Initiative" targeted a 20\% reduction in commercial building energy consumption by 2020 through cost-effective retrofit interventions [6]; similar efforts by the American Institute of Architects [7] and others also promoted savings of $20 \%$ in existing commercial buildings. Several studies aimed to find the cost savings and energy savings potential for energy efficiency retrofits (EERs) in commercial buildings (e.g., [8-16]). EERs may be the most feasible and cost-effective method to improve building energy efficiency [17]. Additionally, due to the large number of older buildings with out-of-date facilities already due for upgrades (many of which are small), there is a vast opportunity to improve the energy efficiency of such building through EER [2]. However, while retrofit may be the most cost-effective option for buildings needing repair, investing in energy efficiency during the retrofit process may still be challenging due to budget constraints or a lack of understanding about the benefits of such retrofits [18]; moreover, there is not a "one size fits all solution" for buildings, particularly small commercial buildings that tend to be more diverse than larger buildings (which are also newer) [19]. Research further documents that building decision-makers have to consider multiple criteria (such as financial decisions, energy consumption, and other decision criteria), and, as such, are faced with a multi-objective optimization problem when determining what criteria to focus on to find solutions that have priority for their building [13,20,21]. Finally, Granderson et al. suggested that perhaps one-quarter of small commercial building owners are motivated to reduce energy use, and a larger segment is aware of potential savings but is wary of making investments [22]. Ultimately, research identifies that there is a great potential for energy efficiency (and, as such, there is a large market for EER construction), but there is a need for user-friendly tools to promote decision makers' ability to justify EER investment for their buildings. Although EER research clearly indicates that EER can be cost-effective, it also points to barriers to EER implementation. However, prior EER research does not identify or explain what motivates decision makers to commit to EER of their buildings. This paper addresses this gap by explicitly identifying the decision criteria used for each case study presented.

\subsection{Barriers to Energy Efficiency Retrofit Implementation}

In addition to documenting the case for EER, previous research documents barriers to achieving energy efficiency in the small commercial buildings market. Jakob found that the biggest challenge that EER projects, both small and large, face is the difficulty to identify the optimal set of solutions for a specific project, due to a lack of understanding of the relationships and synergies among energy efficiency measures, cost, and comfort [23]. Chung et al. studied supermarkets as a proxy for commercial buildings and sought to develop a benchmarking tool for commercial building stock. They discovered that the regression model required to adequately predict performance and support benchmarking involved so many inputs that it became prohibitive to implementation by potential users [24]. Research also highlights the fact that small commercial buildings rarely have a dedicated facilities manager that supervises building operations; as such, the firms that occupy these buildings tend to have lower awareness of their energy use than those in larger commercial buildings (e.g., [25-28]). Moreover, the savings potential in small commercial buildings, in absolute dollars, is lower than their large commercial building counterparts, which often translates to limited rebates and incentives offered for buildings in this segment [28]. Miller et al. found that while government and larger corporate tenants valued 
sustainability and identified it as a critical and non-negotiable criterion when choosing a building, smaller organizations viewed cost and location as the dominant factors and did not consider sustainability when choosing a building. In fact, the smaller organizations included in the study said cost was the greatest barrier they had to sustainability and wanted to see a detailed cost-benefit analysis and payback calculation before adopting an EER [29]. Alhourani and Saxena further report that small business owners themselves seem considerably more likely to implement energy retrofits with payback periods of one year or less [30]. Furthermore, Granderson et al. identified lowering transaction costs as critical for the adoption of energy management in the small commercial building sector, as lower transaction costs make energy retrofits more attractive to small commercial building owners. That study also identified that standardization in data access for utility energy-use data could reduce barriers to adopt energy efficiency measures [22]. Finally, Barnes and Parrish elaborate on additional barriers in this market, including a lack of targeted resources and a lack of financing options for the small commercial market, to name a few [31].

Of the building stock in the commercial building sector, small commercial buildings are an untapped resource for reducing energy consumption in the United States. However, the aforementioned barriers may prevent small commercial building owners from implementing EERs. This paper seeks to address these barriers, and support increased EER implementation in small commercial buildings, by presenting empirical evidence about the efficacy of EERs in the small commercial market, and highlighting why decision-makers chose to implement EERs in this market.

\section{Materials and Methods}

The objective of this study is to determine why small commercial building decisionmakers implement energy efficiency retrofits, and determine the efficacy of various EEMs in these retrofits; taken together, such data can inform strategies to increase retrofits in the small commercial market, as well as which EEMs should be implemented. In order to achieve this objective, the authors searched for small commercial energy efficiency retrofit case studies that included decision criteria, EEMs installed, and verified energy savings. Figure 1 illustrates the case study collection and analysis process.
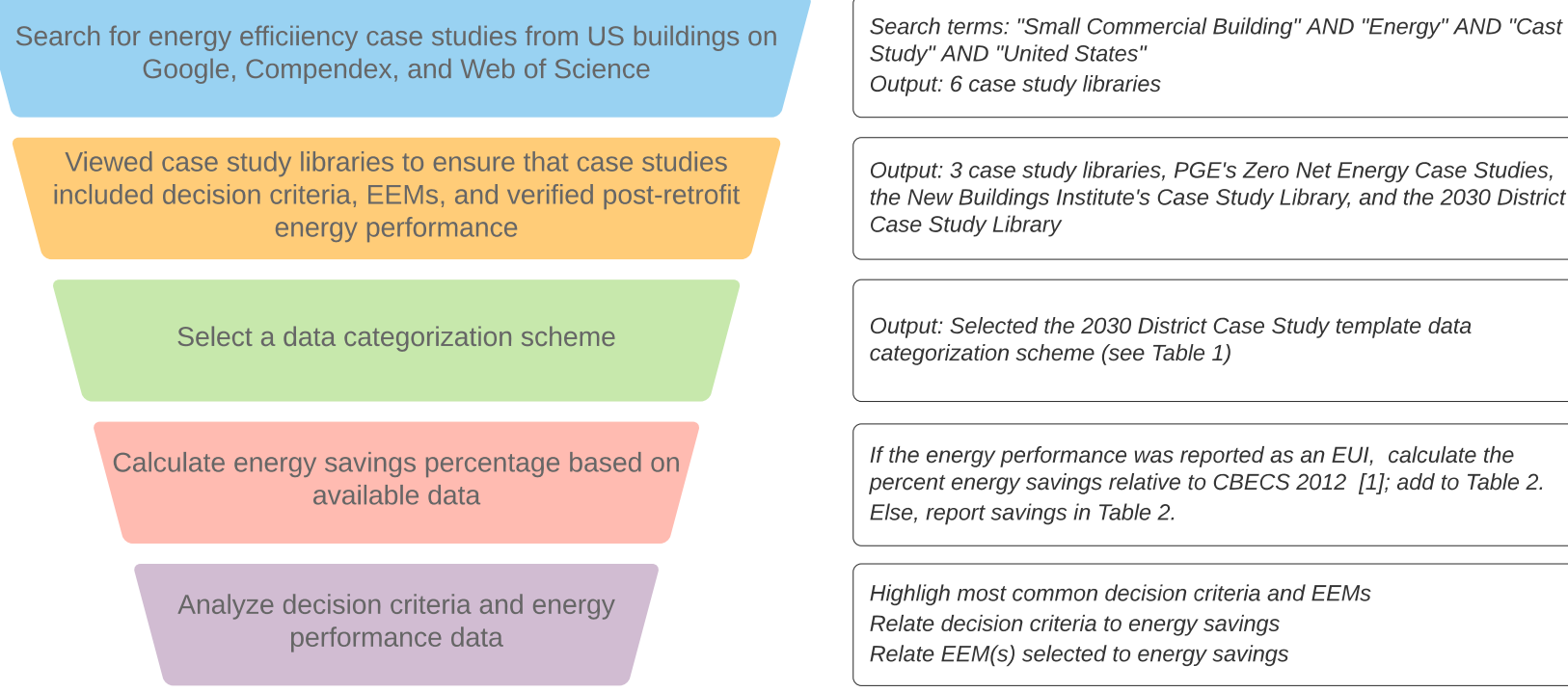

Output: 3 case study libraries, PGE's Zero Net Energy Case Studies, the New Buildings Institute's Case Study Library, and the 2030 District Case Study Library

Output: Selected the 2030 District Case Study template data categorization scheme (see Table 1)

If the energy performance was reported as an EUI, calculate the percent energy savings relative to CBECS 2012 [1]; add to Table 2. Else, report savings in Table 2.

Highligh most common decision criteria and EEMs Relate decision criteria to energy savings Relate EEM(s) selected to energy savings

Figure 1. Research study methodology.

It is noteworthy that, in Figure 1, some case studies collected reported energy savings relative to pre-retrofit energy use, while others reported a post-retrofit EUI. In order to make the data suitable for cross-case study comparison, the authors opt to report all energy performance as an energy savings percentage. Figure 1 also discusses the "data 
categorization scheme", which refers to a standardized approach for reporting decision criteria and EEMs. The authors noticed that each case study library searched had slightly different language for reporting decision criteria and EEMs. For instance, some case studies referenced "End of Life" as a motivation for undertaking a retrofit project, while others referred to "End of Useful Life", and others referred to "Operational". In the authors' read of the case studies, these words all meant the same thing-small commercial building owners opted to implement an energy efficiency retrofit because they would (soon) need to replace equipment anyway ("System Failure" in Table 1 may seem closer to end of life than "Operational", but system failure was more often used to describe inoperable conditions for equipment or systems). Table 1 lists the possible decision criteria and possible EEMs, as well as other options; these were provided by the 2030 District case study template (as described in [31,32]). In Table 1, note that Cooling-Equipment Upgrade/Replacement is listed separately than Cooling-Pumps, despite the fact that pumps can be a piece of cooling equipment when water is the heat exchange medium. This separation was created to explore whether or not ground source heat pumps were used for cooling purposes. In making a specific category, Cooling-Pumps, the intention of the case study developers was to explore the use of heat pump technology for cooling in the small commercial market. The authors use "Cooling-Pumps" throughout the manuscript to maintain consistency with the EEM categorization provided in the 2030 Districts case study portal [32]. Readers should interpret "Cooling-Pumps" as ground source heat pumps.

Table 1. Data categorization scheme (from [32]) that lists options for various case study characteristics.

\begin{tabular}{|c|c|c|c|c|}
\hline Building Type ${ }^{1}$ & EEMs & Decision Criteria & Ownership Model & Financing ${ }^{1}$ \\
\hline Education & $\begin{array}{l}\text { Cooling-Equipment } \\
\text { Upgrade/Replacement }\end{array}$ & Asset Value & Owner Occupied & Self-Financed \\
\hline Food Sales & Cooling_Pumps ${ }^{2}$ & Corporate Values & $\begin{array}{c}\text { Leased/Rent-Owner } \\
\text { Managed }\end{array}$ & Bond Financing \\
\hline Food Service & $\begin{array}{l}\text { DHW-Equipment } \\
\text { Upgrade/Replacement }\end{array}$ & Energy Cost Savings & $\begin{array}{c}\text { Leased/Rent-Non-Owner } \\
\text { Managed }\end{array}$ & $\begin{array}{c}\text { Unsecured Business } \\
\text { Loan }\end{array}$ \\
\hline Office & $\begin{array}{l}\text { Heating-Equipment } \\
\text { Upgrade/Replacement }\end{array}$ & Occupant Comfort & & $\begin{array}{c}\text { Real Estate Backed } \\
\text { Loan }\end{array}$ \\
\hline Public Assembly & $\begin{array}{l}\text { Lighting-Fixture } \\
\text { Upgrade/Replacement }\end{array}$ & Operational & & Crowd Sourced \\
\hline $\begin{array}{c}\text { Public Order and } \\
\text { Safety }\end{array}$ & Lighting-Control & Policy & & Other \\
\hline Mercantile & $\begin{array}{l}\text { Plug Load-Equipment } \\
\text { Upgrade/Replacement }\end{array}$ & $\begin{array}{l}\text { Rebates and } \\
\text { Incentives }\end{array}$ & & \\
\hline \multirow[t]{2}{*}{$\begin{array}{l}\text { Multi-Family } \\
\text { Residential }\end{array}$} & $\begin{array}{c}\text { Plug Load-Management } \\
\text { Strategy }\end{array}$ & System Failure & & \\
\hline & $\begin{array}{c}\text { Plug Load-Commercial } \\
\text { Equipment } \\
\text { Upgrade/Replacement } \\
\text { Ventilation-Air Sealing } \\
\text { Ventilation-Control } \\
\text { Ventilation-Insulation } \\
\text { Ventilation-Openings } \\
\text { Other }\end{array}$ & $\begin{array}{c}\text { Tenant Retention } \\
\text { Other }\end{array}$ & & \\
\hline
\end{tabular}

${ }^{1}$ Column only lists values reflected in the case studies included in this paper. ${ }^{2}$ This is more commonly referred to as a ground source heat pump. However, to maintain consistency with the categorization found in [32], the authors list "Cooling-Pumps" in the table.

\section{Results}

Table 2 presents the results of the data collection process described in Figure 1.

Although Table 2 provides a snapshot of the data collected as part of this study, the authors encourage interested readers to refer to the Supplementary Materials for this paper to see a more complete listing of data about the case studies, including the ownership model and financing type. 
Table 2. Case studies included in this paper.

\begin{tabular}{|c|c|c|c|c|c|}
\hline Case Study & $\begin{array}{c}\text { Energy } \\
\text { Savings (\%) }\end{array}$ & Building Type & Climate & EEMs Implemented & Decision Criteria \\
\hline $1 * *[33]$ & 38 & Education & $\begin{array}{l}\text { 5A-Cool } \\
\text { (Humid) }\end{array}$ & $\begin{array}{l}\text { Lighting-Control } \\
\text { Lighting-Fixture } \\
\text { Upgrade/Replacement } \\
\text { Heating-Equipment } \\
\text { Upgrade/Replacement } \\
\text { Ventilation-Insulation }\end{array}$ & $\begin{array}{l}\text { 1. Corporate Values } \\
\text { 2. Operational }\end{array}$ \\
\hline $2 * *[34]$ & 35 & Office & $\begin{array}{l}\text { 4A-Mixed } \\
\text { (Humid) }\end{array}$ & $\begin{array}{l}\text { Ventilation-Insulation } \\
\text { Ventilation-Control } \\
\text { Lighting-Fixture } \\
\text { Upgrade/Replacement } \\
\text { Heating-Equipment } \\
\text { Upgrade/Replacement }\end{array}$ & $\begin{array}{l}\text { 1. Corporate Values } \\
\text { 2. Energy Cost Savings }\end{array}$ \\
\hline $3 * *[35]$ & 30 & Office & $\begin{array}{l}\text { 7A-Very Cold } \\
\text { (Humid) }\end{array}$ & $\begin{array}{c}\text { Ventilation-Insulation } \\
\text { Ventilation-Openings } \\
\text { Heating-Equipment } \\
\text { Upgrade/Replacement }\end{array}$ & $\begin{array}{l}\text { 1. Energy Cost Savings } \\
\text { 2. Operational }\end{array}$ \\
\hline $4 * *[36]$ & 40 & Office & $\begin{array}{l}5 \mathrm{~A}-\mathrm{Cool} \\
\text { (Humid) }\end{array}$ & $\begin{array}{l}\text { Cooling-Equipment } \\
\text { Upgrade/Replacement } \\
\text { Ventilation-Insulation }\end{array}$ & $\begin{array}{l}\text { 1. Corporate Values } \\
\text { 2. Energy Cost Savings }\end{array}$ \\
\hline $5 * *[37]$ & 29 & Office & $\begin{array}{l}5 \mathrm{~A}-\mathrm{Cool} \\
\text { (Humid) }\end{array}$ & $\begin{array}{l}\text { Ventilation-Insulation } \\
\text { Ventilation-Control } \\
\text { Lighting-Fixture } \\
\text { Upgrade/Replacement } \\
\text { Lighting-Control }\end{array}$ & $\begin{array}{l}\text { 1. Corporate Values } \\
\text { 2. Energy Cost Savings }\end{array}$ \\
\hline 6 ** [38] & 51 & Office & $\begin{array}{l}\text { 3B-Warm } \\
\text { (Dry) }\end{array}$ & $\begin{array}{l}\text { Lighting-Fixture } \\
\text { Upgrade/Replacement } \\
\text { Lighting-Control } \\
\text { Heating-Equipment } \\
\text { Upgrade/Replacement }\end{array}$ & $\begin{array}{l}\text { 1. Corporate Values } \\
\text { 2. Energy Cost Savings }\end{array}$ \\
\hline $7^{* *}[39]$ & 51 & Office & 6B-Cold (Dry) & $\begin{array}{l}\text { Cooling-Equipment } \\
\text { Upgrade/Replacement } \\
\text { Ventilation-Insulation } \\
\text { Lighting-Fixture } \\
\text { Upgrade/Replacement } \\
\text { Heating-Equipment } \\
\text { Upgrade/Replacement }\end{array}$ & $\begin{array}{l}\text { 1. Corporate Values } \\
\text { 2. Energy Cost Savings }\end{array}$ \\
\hline $8 * *[40]$ & 57 & Office & $\begin{array}{l}\text { 4C-Mixed } \\
\text { (Marine) }\end{array}$ & $\begin{array}{c}\text { Lighting-Fixture } \\
\text { Upgrade/Replacement } \\
\text { Lighting-Control } \\
\text { Cooling-Equipment } \\
\text { Upgrade/Replacement } \\
\text { Plug Load-Management Strategy }\end{array}$ & $\begin{array}{l}\text { 1. Corporate Values } \\
\text { 2. Occupant Comfort }\end{array}$ \\
\hline $9^{* *}[41]$ & 69 & Office & $\begin{array}{l}\text { 4C-Mixed } \\
\text { (Marine) }\end{array}$ & $\begin{array}{l}\text { Lighting-Fixture } \\
\text { Upgrade/Replacement } \\
\text { Lighting-Control } \\
\text { Heating-Equipment } \\
\text { Upgrade/Replacement } \\
\text { Plug Load-Equipment } \\
\text { Upgrade/Replacement }\end{array}$ & $\begin{array}{l}\text { 1. Energy Cost Savings } \\
\text { 2. Occupant Comfort }\end{array}$ \\
\hline
\end{tabular}


Table 2. Cont.

\begin{tabular}{|c|c|c|c|c|c|}
\hline Case Study & $\begin{array}{c}\text { Energy } \\
\text { Savings (\%) }\end{array}$ & Building Type & Climate & EEMs Implemented & Decision Criteria \\
\hline $10^{* *}[42]$ & 66 & Office & 6B-Cold (Dry) & $\begin{array}{l}\text { Ventilation-Insulation } \\
\text { Ventilation-Control } \\
\text { Lighting-Fixture } \\
\text { Upgrade/Replacement } \\
\text { Heating-Equipment } \\
\text { Upgrade/Replacement }\end{array}$ & $\begin{array}{l}\text { 1. Other-Family History } \\
\text { 2. Corporate Values }\end{array}$ \\
\hline $11^{* *}[43]$ & 55 & Office & 5B-Cool (Dry) & $\begin{array}{l}\text { Ventilation-Insulation } \\
\text { Ventilation-Control } \\
\text { Lighting-Fixture } \\
\text { Upgrade/Replacement } \\
\text { Lighting-Control }\end{array}$ & $\begin{array}{l}\text { 1. Corporate Values } \\
\text { 2. Energy Cost Savings }\end{array}$ \\
\hline $12[44]$ & 4 & Food Sales & $\begin{array}{l}\text { 4C-Mixed } \\
\text { (Marine) }\end{array}$ & $\begin{array}{l}\text { Lighting-Fixture } \\
\text { Upgrade/Replacement }\end{array}$ & $\begin{array}{l}\text { 1. Rebates and Incentives } \\
\text { 2. Energy Cost Savings } \\
\text { 3. Operational }\end{array}$ \\
\hline $13[45]$ & 6 & Mercantile & $\begin{array}{l}\text { 4C-Mixed } \\
\text { (Marine) }\end{array}$ & $\begin{array}{l}\text { Cooling-Equipment } \\
\text { Upgrade/Replacement }\end{array}$ & $\begin{array}{l}\text { 1. Rebates and Incentives } \\
\text { 2. Energy Cost Savings } \\
\text { 3. Operational }\end{array}$ \\
\hline $14[46]$ & $\mathrm{N} / \mathrm{A}$ & Office & $\begin{array}{l}\text { 4C-Mixed } \\
\text { (Marine) }\end{array}$ & $\begin{array}{l}\text { Lighting-Fixture } \\
\text { Upgrade/Replacement }\end{array}$ & $\begin{array}{ll}\text { 1. } & \text { Energy Cost Savings } \\
\text { 2. } & \text { Operational } \\
\text { 3. } & \text { Other-Aesthetics }\end{array}$ \\
\hline 15 [47] & 10 & Office & $\begin{array}{l}\text { 4C-Mixed } \\
\text { (Marine) }\end{array}$ & $\begin{array}{l}\text { Lighting-Fixture } \\
\text { Upgrade/Replacement }\end{array}$ & $\begin{array}{l}\text { 1. Energy Cost Savings } \\
\text { 2. Operational } \\
\text { 3. Other-Aesthetics }\end{array}$ \\
\hline $16[48]$ & 11 & Food Sales & $\begin{array}{l}\text { 4C-Mixed } \\
\text { (Marine) }\end{array}$ & $\begin{array}{l}\text { Lighting-Fixture } \\
\text { Upgrade/Replacement }\end{array}$ & $\begin{array}{l}\text { 1. Rebates and Incentives } \\
\text { 2. Energy Cost Savings } \\
\text { 3. Operational }\end{array}$ \\
\hline 17 [49] & 19 & Office & $\begin{array}{l}\text { 4C-Mixed } \\
\text { (Marine) }\end{array}$ & $\begin{array}{l}\text { Lighting-Fixture } \\
\text { Upgrade/Replacement }\end{array}$ & $\begin{array}{ll}\text { 1. } & \text { Energy Cost Savings } \\
\text { 2. } & \text { Tenant Retention } \\
\text { 3. } & \text { Occupant Comfort }\end{array}$ \\
\hline $18[50]$ & 10 & Mercantile & $\begin{array}{l}\text { 4C-Mixed } \\
\text { (Marine) }\end{array}$ & $\begin{array}{c}\text { Lighting-Fixture } \\
\text { Upgrade/Replacement }\end{array}$ & $\begin{array}{l}\text { 1. Energy Cost Saving } \\
\text { 2. Environment }\end{array}$ \\
\hline 19 [51] & 8 & Food Sales & $\begin{array}{l}5 \mathrm{~A}-\mathrm{Cool} \\
\text { (Humid) }\end{array}$ & $\begin{array}{l}\text { Lighting-Fixture } \\
\text { Upgrade/Replacement }\end{array}$ & $\begin{array}{l}\text { 1. Energy Cost Saving } \\
\text { 2. System Failure }\end{array}$ \\
\hline $20[52]$ & 14 & Office & $\begin{array}{l}\text { 5A-Cool } \\
\text { (Humid) }\end{array}$ & $\begin{array}{c}\text { Lighting-Fixture } \\
\text { Upgrade/Replacement }\end{array}$ & $\begin{array}{l}\text { 1. Occupant Comfort } \\
\text { 2. Energy Cost Savings }\end{array}$ \\
\hline $21[53]$ & -3 & Food Sales & $\begin{array}{l}\text { 5A-Cool } \\
\text { (Humid) }\end{array}$ & $\begin{array}{l}\text { Lighting-Fixture } \\
\text { Upgrade/Replacement }\end{array}$ & 1. Rebates and Incentives \\
\hline $22[54]$ & 42 & Office & $\begin{array}{l}5 \mathrm{~A}-\mathrm{Cool} \\
\text { (Humid) }\end{array}$ & $\begin{array}{c}\text { Lighting-Fixture } \\
\text { Upgrade/Replacement }\end{array}$ & $\begin{array}{l}\text { 1. Energy Cost Savings } \\
\text { 2. Other-Aesthetics }\end{array}$ \\
\hline $23[55]$ & 23 & $\begin{array}{l}\text { Multi-Family } \\
\text { Residential }\end{array}$ & $\begin{array}{l}\text { 5A-Cool } \\
\text { (Humid) }\end{array}$ & $\begin{array}{c}\text { Lighting-Fixture } \\
\text { Upgrade/Replacement }\end{array}$ & 1. Energy Cost Savings \\
\hline $24[56]$ & 13 & Service & $\begin{array}{l}\text { 3C-Warm } \\
\text { (Marine) }\end{array}$ & $\begin{array}{l}\text { Lighting-Fixture } \\
\text { Upgrade/Replacement }\end{array}$ & 3. Operational \\
\hline $25[57]$ & 24 & $\begin{array}{l}\text { Public } \\
\text { Assembly }\end{array}$ & $\begin{array}{l}\text { 3C-Warm } \\
\text { (Marine) }\end{array}$ & $\begin{array}{l}\text { Lighting-Fixture } \\
\text { Upgrade/Replacement }\end{array}$ & $\begin{array}{l}\text { 1. Energy Cost Savings } \\
\text { 2. Other-Reduced } \\
\text { maintenance }\end{array}$ \\
\hline $26[58]$ & 7 & $\begin{array}{l}\text { Public Order } \\
\text { and Safety }\end{array}$ & $\begin{array}{l}\text { 3C-Warm } \\
\text { (Marine) }\end{array}$ & $\begin{array}{l}\text { Cooling_Equipment } \\
\text { Upgrade/Replacement }\end{array}$ & $\begin{array}{ll}\text { 1. } & \text { Operational } \\
\text { 2. Energy Cost Savings } \\
\text { 3. }\end{array}$ \\
\hline
\end{tabular}


Table 2. Cont.

\begin{tabular}{|c|c|c|c|c|c|}
\hline Case Study & $\begin{array}{c}\text { Energy } \\
\text { Savings (\%) }\end{array}$ & Building Type & Climate & EEMs Implemented & Decision Criteria \\
\hline 27 [59] & 41 & Service & $\begin{array}{l}\text { 3C-Warm } \\
\text { (Marine) }\end{array}$ & $\begin{array}{l}\text { Lighting-Control } \\
\text { Ventilation-Control }\end{array}$ & $\begin{array}{l}\text { 1. Energy Cost Savings } \\
\text { 2. Operational }\end{array}$ \\
\hline $28[60]$ & 3 & $\begin{array}{c}\text { Public } \\
\text { Assembly }\end{array}$ & $\begin{array}{l}\text { 3C-Warm } \\
\text { (Marine) }\end{array}$ & $\begin{array}{l}\text { Cooling-Equipment } \\
\text { Upgrade/Replacement }\end{array}$ & $\begin{array}{l}\text { 1. Energy Cost Savings } \\
\text { 2. Operational }\end{array}$ \\
\hline $29 * *[61]$ & 58 & Office & $\begin{array}{l}\text { 3C-Warm } \\
\text { (Marine) }\end{array}$ & $\begin{array}{c}\text { Lighting-Fixture } \\
\text { Upgrade/Replacement } \\
\text { Lighting-Control } \\
\text { Cooling-Equipment } \\
\text { Upgrade/Replacement } \\
\text { Plug Load-Management Strategy }\end{array}$ & $\begin{array}{l}\text { 1. Corporate Values } \\
\text { 2. Occupant Comfort }\end{array}$ \\
\hline $30 * *[62]$ & 72 & Education & $\begin{array}{l}\text { 3B-Warm } \\
\text { (Dry) }\end{array}$ & $\begin{array}{c}\text { Lighting-Fixture } \\
\text { Upgrade/Replacement } \\
\text { Cooling-Equipment } \\
\text { Upgrade/Replacement } \\
\text { Plug Load-Management Strategy }\end{array}$ & $\begin{array}{l}\text { 1. Rebates and Incentives } \\
\text { 2. Occupant Comfort } \\
\text { 3. System Failure }\end{array}$ \\
\hline $31^{* *}[63]$ & 59 & Office & $\begin{array}{l}\text { 3C-Warm } \\
\text { (Marine) }\end{array}$ & $\begin{array}{c}\text { Lighting-Fixture } \\
\text { Upgrade/Replacement } \\
\text { Lighting-Control } \\
\text { Cooling-Equipment } \\
\text { Upgrade/Replacement } \\
\text { Plug Load-Management Strategy } \\
\text { Ventilation-Controls } \\
\text { Ventilation-Insulation }\end{array}$ & $\begin{array}{ll}\text { 1. } & \text { Policy } \\
\text { 2. } & \text { Operational } \\
\text { 3. } & \text { Energy Cost Savings } \\
\text { 4. } & \text { Asset Value }\end{array}$ \\
\hline $32 * *[64]$ & 75 & Office & $\begin{array}{l}\text { 3B-Warm } \\
\text { (Dry) }\end{array}$ & $\begin{array}{c}\text { Lighting-Fixture } \\
\text { Upgrade/Replacement } \\
\text { Lighting-Control } \\
\text { Cooling-Equipment } \\
\text { Upgrade/Replacement } \\
\text { Plug Load-Management Strategy } \\
\text { Ventilation-Openings } \\
\text { Ventilation-Insulation } \\
\text { Ventilation-Control } \\
\text { DHW-Equipment } \\
\text { Upgrade/Replacement }\end{array}$ & $\begin{array}{l}\text { 1. Asset Value } \\
\text { 2. Rebates and Incentives }\end{array}$ \\
\hline $33^{* *}[65]$ & 67 & Office & $\begin{array}{l}\text { 3C-Warm } \\
\text { (Marine) }\end{array}$ & $\begin{array}{l}\text { Lighting-Fixture } \\
\text { Upgrade/Replacement } \\
\text { Lighting-Control } \\
\text { Cooling-Equipment } \\
\text { Upgrade/Replacement } \\
\text { Plug Load-Equipment } \\
\text { Upgrade/Replacement } \\
\text { Ventilation-Insulation } \\
\text { Ventilation-Controls } \\
\text { DHW-Equipment } \\
\text { Upgrade/Replacement }\end{array}$ & $\begin{array}{l}\text { 1. Corporate Values } \\
\text { 2. Operational } \\
\text { 3. Other-Demonstration } \\
\text { Space }\end{array}$ \\
\hline $34^{* *}[66]$ & 28 & Office & $\begin{array}{l}\text { 3B-Warm } \\
\text { (Dry) }\end{array}$ & $\begin{array}{c}\text { Lighting-Fixture } \\
\text { Upgrade/Replacement } \\
\text { Lighting-Control } \\
\text { Cooling-Equipment } \\
\text { Upgrade/Replacement } \\
\text { Plug Load-Management Strategy }\end{array}$ & $\begin{array}{l}\text { 1. Policy } \\
\text { 2. System Failure } \\
\text { 3. Other-Demonstration } \\
\text { Space }\end{array}$ \\
\hline
\end{tabular}


Figure 2 shows the frequency of decision criteria used, and Figure 3 shows the frequency of various EEMs implemented. For both figures, note that a given case study can employ multiple decision criteria and multiple EEMs, so the frequencies will sum to greater than thirty-four (total number of case studies).

Finally, this paper seeks to provide empirical evidence about energy savings as they relate to decision criteria and EEMs implemented. Figure 4 illustrates this relationship. Though not a perfect metric, the $\mathrm{x}$-axis of Figure 4 lists the number of building systems impacted by EEMs. This is a proxy for the level of integration of the retrofit, with more systems impacted indicating a more integrated package of EEMs, as compared to a single system being impacted, which often correlates to a single EEM being implemented. Similarly, the y-axis explores the energy savings, reported as a percentage. However, in some cases, this percentage is relative to the building's pre-retrofit performance, and in other cases, this is relative to the CBECS average for the given building type (i.e., [1]). Although it would undoubtedly be preferable to report savings relative to a consistent baseline, this was not possible given the data reported. In some cases, the case study listed a post-retrofit EUI, while in other cases, an energy savings was reported relative to pre-retrofit energy consumption. In most cases, it appears that the reason for this difference is the availability of pre-retrofit consumption data, where case studies that do not list pre-retrofit energy consumption report a post-retrofit EUI, and case studies that report an energy savings do not list a post-retrofit EUI, thus making it impossible to compare case studies relative to the same baseline. Nonetheless, the authors feel that this figure adds value as it does provide some insight into the empirical relationship between energy savings and single vs. packaged EEMs.

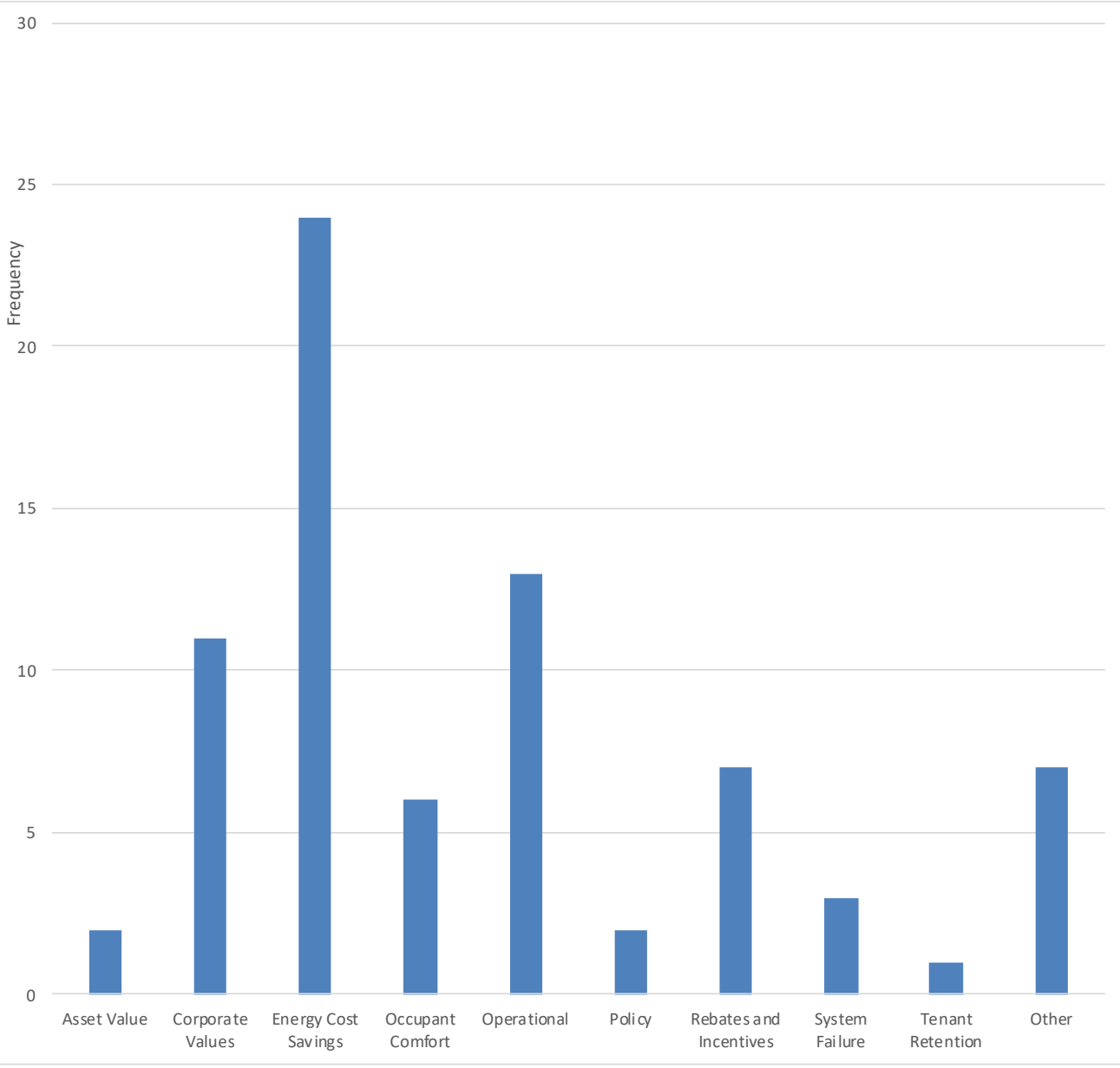

Figure 2. Frequency for decision criteria for the case studies in Table 2. 


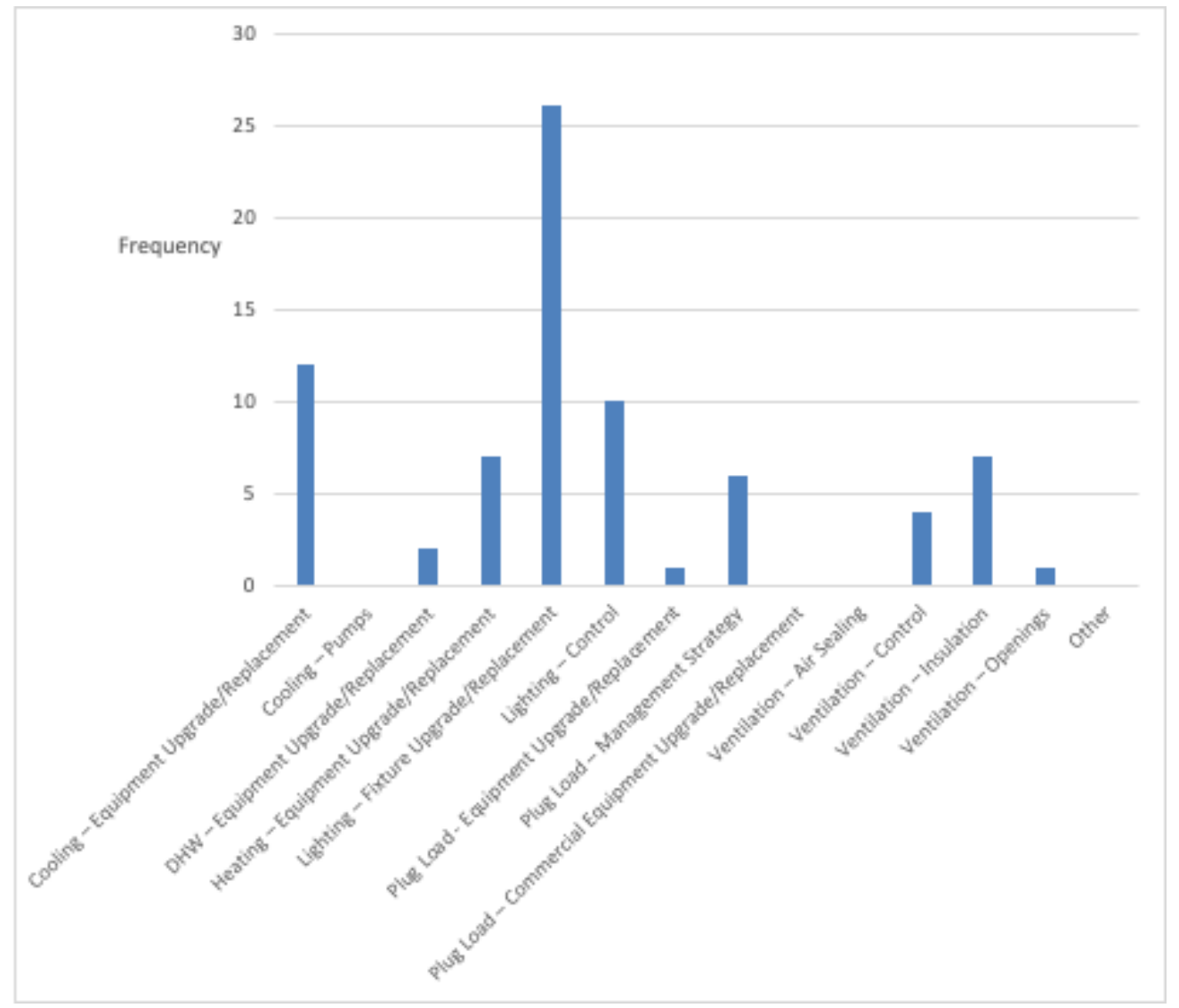

Figure 3. EEM frequency for the case studies in Table 2.

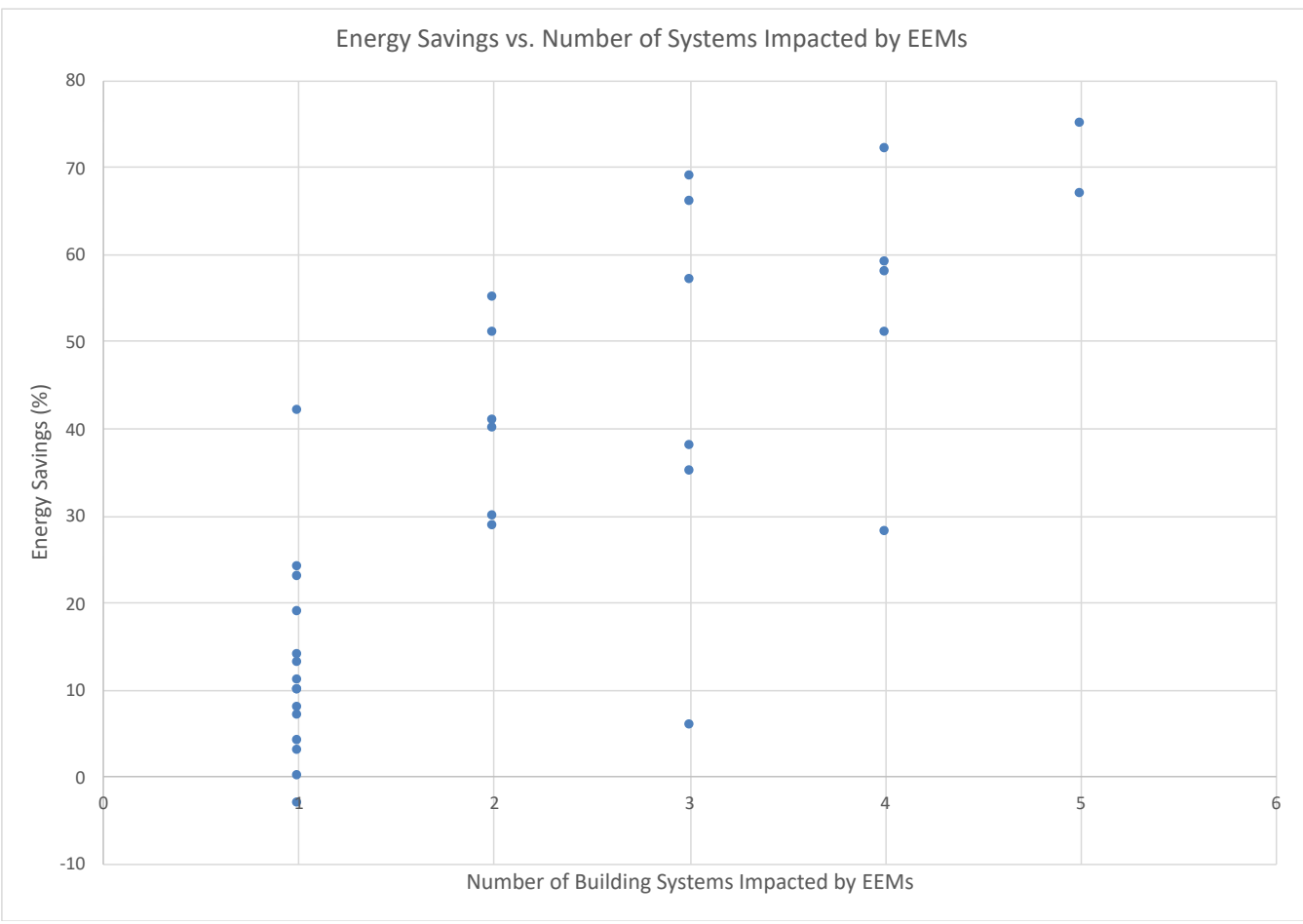

Figure 4. Energy savings for single EEMs vs. integrated EEM packages. 


\section{Discussion}

This section discusses the results of the authors' case study analysis in the context of prior research, as well as in terms of supporting the authors' objective of increasing the number of small commercial building energy efficiency retrofits completed. The section is organized according to the contributions to knowledge stated in the abstract.

\subsection{Decision Criteria}

Figure 2 presents the decision criteria that motivated decision-makers in the small commercial buildings market to implement an energy retrofit in their building (or space). By a considerable margin, decision-makers were motivated by energy cost savings potential associated with implementing a retrofit. Operational considerations followed, and rebates and incentives rounded out the top three decision criteria. These findings essentially reinforce what was already known about this market: cost savings drive decision-making, likely because many small commercial building owners and tenants are also small business owners, so to invest capital, the decision-maker needs to be confident in the associated return.

The fact that operational criteria was the second most-cited decision criteria provides insight into how to expand the prevalence of energy efficiency retrofits in this market. In particular, operational denotes ease of maintenance, and may also indicate that an energy retrofit decision was made because a piece of equipment (or pieces) was nearing its end of life, so there was a "natural" opportunity to improve energy performance ("System Failure" denotes that equipment has become non-functional, not that it will soon become non-functional). This finding reinforces the work of Mathew et al. [19] and Granderson et al. [22]; these studies suggest that end of equipment life is an opportunity to engage building owners in a conversation about improving energy efficiency. Indeed, one of the cases studied, a California office building, highlights how cost-effective energy efficiency retrofits look when there is already some level of retrofit required, particularly compared to simply taking on an energy retrofit only for the sake of energy savings [64]. Indeed, another case study documents the owner's unwillingness to replace equipment before that is required [53]. Notably, food service owners report that they prefer to replace equipment prior to failure so as not to lose operating time for their business; this owner indicated that the increased cost for a more efficient freezer was more than offset by energy savings during operations (e.g., [51]).

Finally, Figure 2 illustrates the relative lack of prevalence for decision criteria relating to corporate values, comfort, and policy. This suggests that when marketing to small commercial building decision-makers, arguments about sustainability and energy efficiency are unlikely to be persuasive on their own. Rather, marketing requires making a solid case for the cost savings associated with implementing an energy retrofit, and the results in Table 2 support making such a claim. Nearly every case study resulted in energy savings and in the one case where energy savings were not reported (\#21 in Table 2), the increase in energy consumption is due to increased occupancy in the building, which translated to increased sales, so the owner still reports satisfaction with the energy retrofit.

\subsection{EEMs Implemented in the Small Commercial Buildings Case Studies}

Figure 3 presents the EEMs implemented in the case studies. Somewhat unsurprisingly, lighting retrofits were by far the most commonly implemented in the cases studied. This is reasonable, given the relatively straightforward energy cost calculations associated with fixture replacement. Indeed, a lighting retrofit is a low-risk proposition, not only in terms of costs and resulting payback, but also in terms of disruption to the building occupants during the retrofit. Completing a lighting fixture replacement does not take long, and buildings can typically be occupied throughout the retrofit.

Excepting lighting, cooling equipment replacement or upgrades were the most prevalent. This is likely related to the clear value proposition for such replacement, in terms of cost savings, more reliable cooling, and limiting the risk associated with a cooling equipment failure. The authors note that this EEM selection may also be impacted by the 
climates in the cases studied - in many cases, the cooling load was not dominant, so the risk of failure, while detrimental for a relatively short period of time, would not be as great as the risk of heating system failure.

Finally, there is arguably more to be learned from what was not implemented than from what was implemented. Small commercial building decision-makers did not invest much in plug load or ventilation EEMs, likely because the cost-effectiveness for such EEMs is related to occupant behavior, which can be hard to manage.

Taken together, the EEMs implemented illustrates the need for quick wins in this market. Often, decision-makers opted to only implement a single EEM, which should signal to incentive program developers and others engaged in the small commercial buildings market the need to ensure that the case for an energy retrofit fits naturally into the decision-makers business model. Unsurprisingly, the case studies that implemented packages of EEMs were also cases where the decision-maker was at a natural point of inflection in the real estate life cycle (e.g., renewing a 10-year lease [61], already in the construction process for the retrofit [66], or looking to re-lease a vacant building [64]). This further amplifies the previously discussed findings, that to sell to small commercial buildings, ease of retrofit and reliability of savings is critical.

\subsection{Efficacy of Single EEMs versus EEM Packages}

Figure 4 illustrates that, in general, the more integrated the EEMs implemented, the greater the energy savings. Indeed, with one exception, the lowest savings reported for a given number of systems impacted is higher than the highest savings for the next-lowest number of systems impacted. This supports, with empirical evidence, the relative benefits of integrated EEM approaches as compared to single EEM implementations. Given what has already been discussed about the importance of reliable energy savings (that is, low-risk investments), and the associated energy cost savings, it seems counterintuitive that so few small commercial building decision-makers opted to install integrated EEM packages, rather than single EEMs. After all, if the objective is to reduce energy costs by as much as possible, the easiest way to do this is to implement EEM packages that support greater energy savings. However, a closer analysis of the case studies reveals that integrated EEM packages were installed when the real estate lifecycle supported it or when the building owner was required, by their own mission or by a new policy, to achieve deep energy savings.

Going forward, data such as that presented in Figure 4 should be presented to small commercial building owners when they are planning a retrofit, for whatever reason. If there is already an appetite to invest in the retrofit, then energy efficiency retrofits become a much less risky proposition. Moreover, the non-energy benefits (e.g., [67-70]) can be explained and explored to further the case for an energy efficiency retrofit. Such data can also help utility program developers and small commercial building energy efficiency advocates to craft specific incentive structures for the small commercial buildings market to increase the implementation of EEM packages. In particular, providing design assistance or technical assistance may reduce the burden on small commercial building decision-makers that are interested in achieving deep energy savings, but may lack the upfront capital required to ensure synergistic EEM packages are developed and deployed; indeed, many of the zero net energy retrofits highlighted the need for such technical expertise [61,64-66].

\subsection{Policy Implications and Potential $\mathrm{CO}_{2}$ Reduction}

As energy policy evolves in the United States, the objective of policies that target commercial buildings, regardless of size, seems to be decarbonization (e.g., [71,72]). Indeed, decarbonization remains a critical goal to curb climate change and its impacts on economic, environmental, and social systems worldwide. This research indicates that for small commercial building owners and decision-makers, climate change alone, and by extension, decarbonization, is unlikely to motivate a retrofit. However, policy makers can leverage the results of this paper to develop policies and incentives that do motivate decision-makers 
in the small commercial buildings market. In particular, Figure 2 indicates that saving costs is the most-cited decision criteria for small commercial owners. Policy-makers can approach this in two ways: (1) provide grants or on-bill utility savings to reduce the upfront costs required to reap the desired energy cost savings, or (2) implement energy or carbon budgets and impose fines to those building owners that exceed the budget. Given that small commercial buildings tend to be older, and, therefore, less efficient on average (according to [1]), the latter may pose an inequitable burden on small commercial building owners and tenants. As such, the authors recommend that policy-makers focus on providing funds to small commercial building decision-makers willing to retrofit their buildings, perhaps leveraging a shared savings model (e.g., [16]) to make such a policy revenue-neutral. Were such funds available, the retrofit rate in the small commercial sector may approach the "very aggressive" threshold, at $0.95 \%$ [73], i.e., $0.95 \%$ of the national small commercial building floor area is being retrofit for energy savings annually. Table 3 illustrates annual potential energy and net carbon savings associated with conducting energy retrofits on $0.95 \%$ of the small commercial building stock. The authors explore reductions associated with 5\%,10\%, and 20\% energy savings at the building level, as these savings seem achievable based on Figure 4, depending on the number of building systems impacted by the retrofit project. Table 3 illustrates that even with a "very aggressive" energy retrofit rate of $0.95 \%$, the carbon emissions reductions associated with small commercial building retrofits are relatively small, ranging from $662,000 \mathrm{kgCO}_{2} /$ year for $5 \%$ energy savings to $2,647,000 \mathrm{kgCO}_{2}$ / year for $20 \%$ energy savings. This highlights the critical need for increasing the retrofit rate. Arguably, this increase in retrofit rate is the real driver for policy.

Table 3. Annual energy and net carbon emissions impacts from small commercial building retrofits, assuming a $0.95 \%$ retrofit rate.

\begin{tabular}{|c|c|c|}
\hline & Energy (kWh) & Carbon Emissions ${ }^{1}\left(\mathrm{mtCO}_{2}\right)$ \\
\hline \multirow{2}{*}{$\begin{array}{l}\text { Baseline commercial building consumption } \\
\text { Baseline small commercial consumption } \\
\text { (40\% of total) }\end{array}$} & $4,912,749,173^{2}$ & $3,483,139.2$ \\
\hline & $1,965,099,669.2$ & $1,393,255.7$ \\
\hline Baseline consumption of buildings to be retrofit ${ }^{3}$ & $18,668,446.9$ & $13,235.9$ \\
\hline $\begin{array}{l}\text { Annual Reductions, } \\
5 \% \text { energy savings }\end{array}$ & $933,422.3$ & 661.8 \\
\hline $\begin{array}{l}\text { Annual Reductions, } \\
10 \% \text { energy savings }\end{array}$ & $1,866,844.7$ & 1323.6 \\
\hline $\begin{array}{l}\text { Annual Reductions, } \\
20 \% \text { energy savings }\end{array}$ & $3,733,689.4$ & 2647.2 \\
\hline
\end{tabular}

${ }^{1}$ From https://www.epa.gov/energy/greenhouse-gases-equivalencies-calculator-calculations-and-references (accessed on 25 September 2021). ${ }^{2}$ From https://www.eia.gov/totalenergy/data/monthly/pdf/sec2_7.pdf (accessed on 25 September 2021). ${ }^{3} 95 \%$ of the small commercial consumption, based on $95 \%$ retrofit rate.

Importantly, the authors note that policy need not come from local, state, or federal regulations, it can come in the form of a utility program with on-bill financing that small commercial building owners can access or loans from the Small Business Administration. Indeed, on-bill financing was a popular mechanism for funding lighting retrofits, which remain dominant in the small commercial space (Figure 2). If funds can be accessed, this reduces a critical barrier for small commercial building decision-makers; thus, the objective should then become getting decision-makers to increase the scope of their retrofit to include more than one system and increase energy savings. To achieve this, the authors advocate a different approach: policies and incentive programs may prove instrumental in beginning to engage the small commercial market, and, thus, increasing the retrofit rate. However, increasing the energy savings yielded from a retrofit would be difficult to do from a policy perspective, as there is not a "one size fits all" approach for increasing savings, given the diversity of the building stock and the systems that serve it. 
Indeed, the authors argue that to increase the savings associated with a given project, the focus should not be on an external policy that small commercial building decisionmakers engage; rather, the focus should shift to educating these decision-makers about all of the non-energy benefits of an energy retrofit. The case studies reviewed in this paper are designed to do exactly that: motivate decision-makers directly to take on an energy retrofit in order to reap the non-energy benefits alongside the cost savings. Although the case studies are undeniably compelling, they may not be visible to their target audience, as these studies are housed on websites that likely do not relate to a small commercial building decision-maker's core business. Thus, the authors propose that energy efficiency advocates partner with the small business associations, or trade organizations, that small commercial building owners regularly engage, like a local chamber of commerce. This approach may prove most effective in illustrating the "win-win" nature of an energy retrofit, and the messaging will come from a trusted source. Moreover, this approach will offer an opportunity to engage multiple small commercial building decision-makers simultaneously. Finally, such an approach has the potential to increase the retrofit rate to be higher than $1 \%$, which is certainly required to meet urgent climate action goals. The authors note that the renovation rate, which Coffey et al. define as "buildings being renovated for architectural or function purposes" is $4 \%$ in the "very aggressive" case [73]. Therefore, if these renovations then included energy retrofits, the overall rate of energy retrofits would climb to nearly $5 \%$, meaning that all buildings would have energy retrofits in two decades, which is certainly more encouraging than the ten decades it would take if only energy retrofits were included in the rate.

\section{Conclusions}

This paper presented data from 34 small commercial building energy retrofit case studies in the United States. The data illustrate that in the small commercial market, energy cost savings are the most prevalent decision criteria cited, supporting the notion that energy retrofits are easiest to sell to decision-makers when there is a compelling and reliable case to be made for reducing energy costs. Further, the case studies illustrate that many decision-makers opt to implement a single EEM, or EEMs that only impact a single building system; generally speaking, this takes the form of a lighting retrofit. This is likely related to the first finding; lighting retrofits very reliably save energy and, thus, reduce operating costs. Finally, this paper illustrates the relative benefits of implementing an integrated package of EEMs as compared to implementing a single EEM. The authors hope that this final finding can be leveraged when speaking to decision-makers about the energy cost savings associated with an integrated EEM package to accelerate adoption of such packages in the small commercial buildings market. Indeed, taking an integrated approach to energy retrofits increases the energy efficiency, as well as the carbon savings, thus supporting achieving energy and carbon reduction goals with a single intervention. If small commercial buildings achieve a $20 \%$ energy savings, and $0.95 \%$ of the small commercial stock is retrofit each year, a savings of 2,647,000 $\mathrm{kgCO}_{2} /$ year would result. If the retrofit rate can be increased, the carbon emissions reductions will further increase, highlighting the paramount importance of convincing more small commercial building owners to implement energy retrofits.

Supplementary Materials: The following are available online at https:/ /www.mdpi.com/article/ 10.3390/en14196207/s1, Table S1: Small Commercial Case Studies Meta Analysis.

Author Contributions: Conceptualization, all authors.; methodology, all authors.; validation, R.S. and H.N.; formal analysis, all authors.; investigation, R.S. and H.N.; data curation, all authors; writing-original draft preparation, R.S. and H.N.; writing-review and editing, K.P.; supervision, K.P.; project administration, K.P.; funding acquisition, K.P. All authors have read and agreed to the published version of the manuscript. 
Funding: This research was funded in part by the US Department of Energy, contract number DENL0025552. The views presented in this paper are the views of the authors and do not necessarily reflect the views of the United States Department of Energy.

Data Availability Statement: Publicly available datasets were analyzed in this study. These data can be found in the references cited in Table 2. An expanded view of Table 2 is also available as Supplementary Material.

Acknowledgments: The authors gratefully acknowledge the support of collaborators on the 2030 Small Commercial Districts and Toolkit project; in particular Cindy Regnier, Vincent Martinez, and Keven Settlemyre.

Conflicts of Interest: The authors declare no conflict of interest.

\section{References}

1. U.S. EIA. Commercial Buildings Energy Consumption Survey (CBECS); U.S. Energy Information Administration: Washington, DC, USA, 2021. Available online: https://www.eia.gov/consumption/commercial/data/2012/ (accessed on 30 April 2021).

2. Langner, R.; Hendron, B.; Pless, S.; Huppert, M.; Cochrane, R. Industry Research and Recommendations for Small Buildings and Small Portfolios; NREL/TP-5500-57776; National Renewable Energy Laboratory: Golden, CO, USA, 2013. Available online: https:/ / www.nrel.gov/docs/fy14osti/57776.pdf (accessed on 25 September 2021).

3. Preservation Green Lab and the New Buildings Institute. Realizing the Energy Efficiency Potential of Small Commercial Buildings; Preservation Green Lab and the New Buildings Institute: Washington, DC, USA, 2013. Available online: http:/ / www3.cec.org/ islandora-gb/islandora/object/islandora:1022/datastream/OBJ-EN/view (accessed on 25 September 2021).

4. US DOE. Small Buildings = Big Opportunity for Energy Savings; U.S. Department of Energy: Washington, DC, USA, 2013. Available online: https:/ / www.nrel.gov/docs/fy14osti/60917.pdf (accessed on 30 April 2021).

5. US EIA. U.S. Energy Consumption by Source and Sector, 2019; U.S. Energy Information Administration: Washington, DC, USA, 2019. Available online: https://www.eia.gov/totalenergy/data/monthly/pdf/flow/css_2019_energy.pdf (accessed on 30 April 2021).

6. US DOE. Better Buildings Challenge; U.S. Department of Energy: Washington, DC, USA, 2021. Available online: https:// betterbuildingssolutioncenter.energy.gov/challenge (accessed on 30 April 2021).

7. Architecture 2030. The 2030 Challenge for Planning; Architecture 2030: Seattle, WA, USA, 2015. Available online: http:// architecture2030.org/2030_challenge/2030_challenge_planning (accessed on 19 February 2015).

8. Androutsopoulos, A.V.; Spanou, A. Energy Efficiency Actions to Uptake Energy Retrofitting Measures in Buildings. Proc. Environ. Sci. 2017, 38, 875-881. [CrossRef]

9. Eames, M.; Dixon, T.; May, T.; Hunt, M. City Futures: Exploring Urban Retrofit and Sustainable Transitions. Build. Res. Inf. 2013, 41, 504-516. [CrossRef]

10. Hamoud, M.; McGee, P. Energy Efficient Design \& Retrofit of Boiler \& Steam Generation System. In Proceedings of the 2nd International Conference on the Global Impact of EnMS, Dublin, Ireland, 4 May 2012; Sustainable Energy Authority of Ireland: Dublin, Ireland, 2012.

11. Ladhad, A.; Parrish, K. Phoenix's First Zero-Net Energy Office Retrofit: A Green and Lean Case Study. J. Green Build. 2014, 8, 3-16. [CrossRef]

12. Parrish, K. A Path to Successful Energy Retrofits: Early Collaboration through Integrated Project Delivery Teams; LBNL-6130E; Lawrence Berkeley National Laboratory: Berkeley, CA, USA, 2012. Available online: http://eetd.lbl.gov/sites/all/files/lbnl-6130e.pdf (accessed on 25 September 2021).

13. PNNL. Advanced Energy Retrofit Guides; U.S. Department of Energy: Washington, DC, USA, 2011.

14. Rabani, M.; Madessa, H.B.; Nord, N. A state-of-art review of retrofit interventions in buildings towards nearly zero energy level. Energy Proc. 2017, 134, 317-326. [CrossRef]

15. Rios, F.C.; Parrish, K.; Chong, O. Low Cost Energy Retrofit Method for Small and Medium Commercial Buildings. Proc. Eng. 2016, 145, 172-179. [CrossRef]

16. Sanders, M.; Parrish, K.; Earni, S. Savings to Sustainability: Application of a Novel Approach to Delivering a Sustainable Built Environment. J. Archit. Eng. 2013, 19, 156-163. [CrossRef]

17. Zheng, L.; Lai, J. Environmental and economic evaluations of building energy retrofits: Case study of a commercial building. Build. Environ. 2018, 145, 14-23. [CrossRef]

18. Turnbull, P. Foreward. In Zero Net Energy Case Study Buildings; Dean, E., Ed.; Pacific Gas and Electric (PGE): San Francisco, CA, USA, 2016; Volume 2, pp. 4-5.

19. Mathew, P.; Coleman, P.; Page, J.; Regnier, C.; Shackelford, J.; Jungclaus, M.; Olgyay, V. Energy Efficiency and the Real Estate Lifecycle: Stakeholder Perspectives; LBNL-2001214; Lawrence Berkeley National Laboratory: Berkeley, CA, USA, 2019. Available online: https:/ / eta-publications.lbl.gov/sites/default/files/energy_efficiency_and_the_real_estate_-_paul_mathew.pdf (accessed on 25 September 2021). 
20. Ding, X.; Parish, K. A Proposed Lean Decision-Making Process for Building Energy Retrofits. In Proceedings of the 27th Annual Conference of the International Group for Lean Construction (IGLC), Dublin, Ireland, 3 July 2019; pp. 1357-1366. Available online: http:/ /iglc.net/Papers/Details/1706 (accessed on 25 September 2021). [CrossRef]

21. Askari, N.; Parrish, K. A Novel Process for Selecting a PCM for a Building Energy Retrofit. ASHRAE Trans. 2021, in press.

22. Granderson, J.; Hult, E.; Fernandes, S.; Mathew, P.; Mitchell, R. Unlocking Energy Efficiency in Small Commercial Buildings through Mechanical Contractors. ASCE J. Archit. Eng. 2017, 23, C4016001. [CrossRef]

23. Jakob, M. The Trade-Offs and Synergies between Energy Efficiency, Costs, and Comfort in Office Buildings. In Proceedings of the CISBAT, Lausanne, Switzerland, 4-5 September 2007.

24. Chung, W.; Hui, Y.V.; Lam, Y.M. Benchmarking the energy efficiency of commercial buildings. Appl. Energy 2006, 83, 1-14. [CrossRef]

25. Hong, T.; Piette, M.A.; Chen, Y.; Lee, S.H.; Taylor-Lange, S.C.; Zhang, R.; Sun, K.; Price, P. Commercial Building Energy Saver: An energy retrofit analysis toolkit. Appl. Energy 2015, 159, 298-309. [CrossRef]

26. Lee, S.H.; Hong, T.; Piette, M.A.; Sawaya, G.; Chen, Y.; Taylor-Lange, S.C. Accelerating the energy retrofit of commercial buildings using a database of energy efficiency performance. Energy 2015, 90, 738-747. [CrossRef]

27. Ketchman, K.; Khanna, V.; Parrish, K.; Bilec, M.M. Small Commercial's Big Problem: Disaggregating Whole Building Energy Bills. In Proceedings of the 2017 Joint Conference of ISIE and ISSST, Chicago, IL, USA, 25-29 June 2017.

28. Ketchman, K.J.; Parrish, K.; Khanna, V.; Bilec, M.M. Small Business Electricity Disaggregation: Where can we improve? Towards increased transparency of appliance modal parameters. Energy Build. 2018, 176, 194-202. [CrossRef]

29. Miller, E.; Buys, L. Retrofitting commercial office buildings for sustainability: Tenants' perspectives. J. Prop. Invest. Financ. 2008, 26, 552-561. [CrossRef]

30. Alhourani, F.; Saxena, U. Factors affecting the implementation rates of energy and productivity recommendations in small and medium sized companies. J. Manuf. Syst. 2009, 28, 41-45. [CrossRef]

31. Barnes, E.A.; Parrish, K. Small Buildings, Big Impacts: The Role of Small Commercial Building Energy Efficiency Case Studies in 2030 Districts. Sustain. Cities Soc. 2016, in press. [CrossRef]

32. 2030 Districts. Create a Case Study. Available online: https://www.2030districts.org/node/add/case-studies?field_case_study_ project $=248$ (accessed on 30 April 2021).

33. 2030 Districts. The Barn at Fallingwater. Architecture 2030: Seattle, WA, USA, 2021. Available online: https:/ /www.2030districts. org/case-study/barn-fallingwater (accessed on 30 April 2021).

34. 2030 Districts. Oak Ridge National Laboratory Office Building 3156. Architecture 2030: Seattle, WA, USA, 2021; Available online: https:/ / www.2030districts.org/case-study/oak-ridge-national-laboratory-office-building (accessed on 30 April 2021).

35. 2030 Districts. NOAA's Weather Forecast Office. Architecture 2030: Seattle, WA, USA, 2021. Available online: https://www.2030 districts.org/case-study/noaas-weather-forecast-office-caribou-maine (accessed on 30 April 2021).

36. 2030 Districts. Elevate Energy Building. Architecture 2030: Seattle, WA, USA, 2021. Available online: https://www.2030districts. org/case-study/elevate-energy-building (accessed on 30 April 2021).

37. AIA. CCI Center. The American Institute of Architects. Available online: http://www.aiatopten.org/node/218 (accessed on 30 April 2021).

38. NRDC. Case Study: NRDC Office; Natural Resources Defense Council: New York, NY, USA, 2021. Available online: https://www. nrdc.org/sites/default/files/nrdcsm.pdf (accessed on 30 April 2021).

39. NBI. Case Study, Deep Energy Savings in Existing Buildings: Home on the Range; New Buildings Institute (NBI): Portland, OR, USA, 2021. Available online: http:/ / newbuildings.org/sites/default/files/Case_Study_Home-on-the-Range.pdf (accessed on 30 April 2021).

40. NBI. Case Study, Deep Energy Savings in Existing Buildings: The Lovejoy Building; New Buildings Institute (NBI): Portland, OR, USA, 2021. Available online: https://newbuildings.org/sites/default/files/Case_Study_Lovejoy.pdf (accessed on 30 April 2021).

41. NBI. Case Study, Deep Energy Savings in Existing Buildings: Johnson Braund Design Group; New Buildings Institute (NBI): Portland, OR, USA, 2021. Available online: http://newbuildings.org/sites/default/files/Case_Study_Johnson-Braund-Design-Grp.pdf (accessed on 30 April 2021).

42. NBI. Case Study, Deep Energy Savings in Existing Buildings: The Beardmore Building; New Buildings Institute (NBI): Portland, OR, USA, 2021. Available online: https://newbuildings.org/sites/default/files/Case_Study_Beardmore.pdf (accessed on 30 April 2021).

43. NBI. Case Study, Deep Energy Savings in Existing Buildings: Alliance Center; New Buildings Institute (NBI): Portland, OR, USA, 2021. Available online: http:/ / newbuildings.org/sites/default/files/Case_Study_Alliance-Center.pdf (accessed on 30 April 2021).

44. 2030 Districts. Corner Market, Pikes Place. Architecture 2030: Seattle, WA, USA, 2021. Available online: https://www.2030 districts.org/case-study/pike-place-market-corner-market-lighting-retrofit (accessed on 30 April 2021).

45. 2030 Districts. Main Market, Pikes Place. Architecture 2030: Seattle, WA, USA, 2021. Available online: https://www.2030districts. org/case-study/ pike-place-market-main-market-led-upgrade (accessed on 30 April 2021).

46. 2030 Districts. 6 Leggs LLC \& Rosenast III LLC. Architecture 2030: Seattle, WA, USA, 2021. Available online: https://www.2030 districts.org/case-study /6-leggs-llc-lighting-retrofit (accessed on 30 April 2021).

47. 2030 Districts. Groff and Murphy Law (300 E Pine Street). Architecture 2030: Seattle, WA, USA, 2021. Available online: https:/ / www.2030districts.org/case-study/groff-murphy-300-e-pine-lighting-retrofit (accessed on 30 April 2021). 
48. 2030 Districts. Sanitary Market, Pikes Place. Architecture 2030: Seattle, WA, USA, 2021. Available online: https://www.2030 districts.org/case-study/pike-place-market-sanitary-market-lighting-retrofit (accessed on 30 April 2021).

49. 2030 Districts. 1016 East Pikes Place. Architecture 2030: Seattle, WA, USA, 2021. Available online: https://www.2030districts. org/case-study/ten-sixteen-east-pike-lighting-retrofit (accessed on 30 April 2021).

50. 2030 Districts. 8 Limbs Yoga. Architecture 2030: Seattle, WA, USA, 2021. Available online: https://www.2030districts.org/casestudy/8-limbs-yoga-center-lighting-retrofit (accessed on 30 April 2021).

51. 2030 Districts. Pamela's Diner. Architecture 2030: Seattle, WA, USA, 2021. Available online: https://www.2030districts.org/casestudy/pamelas-diner-refrigeration-replacement-and-lighting-upgrade (accessed on 30 April 2021).

52. 2030 Districts. Neighbourhood Legal Services. Architecture 2030: Seattle, WA, USA, 2021. Available online: https://www.2030 districts.org/case-study/neighborhood-legal-services-association (accessed on 30 April 2021).

53. 2030 Districts. Oakland Real Estate, Dave and Andy's Homemade Ice Cream. Architecture 2030: Seattle, WA, USA, 2021. Available online: https:/ / www.2030districts.org/case-study/dave-andys-ice-cream-shop (accessed on 30 April 2021).

54. 2030 Districts. 900 Penn. Architecture 2030: Seattle, WA, USA, 2021. Available online: https://www.2030districts.org/casestudy/900-penn-ave-trek-development (accessed on 30 April 2021).

55. 2030 Districts. Carlysle Multi-Family. Architecture 2030: Seattle, WA, USA, 2021. Available online: https://www.2030districts. org/case-study/carlyle-common-space (accessed on 30 April 2021).

56. 2030 Districts. Berkeley Transfer Station. Architecture 2030: Seattle, WA, USA, 2021. Available online: https://www.2030districts org/case-study/berkeley-transfer-station (accessed on 30 April 2021).

57. 2030 Districts. Rendezvous, 2320 2nd Ave. Architecture 2030: Seattle, WA, USA, 2021. Available online: https://www.2030 districts.org/case-study/rendezvous-cafe-lighting-retrofit (accessed on 30 April 2021).

58. 2030 Districts. Redwood City Police HQ (High Efficiency Filter Upgrades). Architecture 2030: Seattle, WA, USA, 2021. Available online: https: / /www.2030districts.org/case-study/1301-maple-st-hvac-upgrade (accessed on 30 April 2021).

59. 2030 Districts. City of Berkeley Animal Shelter. Architecture 2030: Seattle, WA, USA, 2021. Available online: https://www.2030 districts.org/case-study/animal-shelter-rcx-3 (accessed on 30 April 2021).

60. 2030 Districts. South San Francisco Conference Center (AHU Replacement Project). Architecture 2030: Seattle, WA, USA, 2021. Available online: https:/ /www.2030districts.org/case-study/south-san-francisco-conference-center (accessed on 30 April 2021).

61. Dean, E. DPR Construction Office Building. In Zero Net Energy Case Study Buildings; Pacific Gas and Electric (PGE): San Francisco, CA, USA, 2016; Volume 2, pp. 2-27.

62. NBI. Emerging Zero Net Energy School Retrofit Case Study: Newcastle Elementary School; New Buildings Institute (NBI): Portland, OR, USA, 2017. Available online: https://newbuildings.org/wp-content/uploads/2018/01/NBI_Case-StudyProp39_Newcastle.pdf (accessed on 30 April 2021).

63. NBI. Emerging Zero Net Energy Building Case Study: California State Lottery Santa Fe Springs District Office; New Buildings Institute (NBI): Portland, OR, USA, 2016. Available online: https://newbuildings.org/wp-content/uploads/2017/03/CPUC_CALottery. pdf (accessed on 30 April 2021).

64. NBI. Verified Zero Net Energy Building Case Study: 435 Indio Way; New Buildings Institute (NBI): Portland, OR, USA, 2016. Available online: https://newbuildings.org/wp-content/uploads/2017/03/CPUC_435Indio.pdf (accessed on 30 April 2021).

65. Dean, E. IBEW-NECA JATC Training Facility. In Zero Net Energy Case Study Buildings; Pacific Gas and Electric (PGE): San Francisco, CA, USA, 2016; Volume 2, pp. 28-49.

66. Dean, E. California DMV Field Office. In Zero Net Energy Case Study Buildings; Pacific Gas and Electric (PGE): San Francisco, CA, USA, 2018; Volume 3, pp. 62-77.

67. Regnier, C.; Sun, K.; Hong, T.; Piette, M.A. Quantifying the benefits of a building retrofit using an integrated system approach: A case study. Energy Build. 2018, 159, 332-345. [CrossRef]

68. Bleyl, J.; Bareit, M.; Casas, M.A.; Chatterjee, S.; Coolen, J.; Hulshoff, A.; Lohse, R.; Mitchell, S.; Robertson, M.; Ürge-Vorsatz, D. Office building deep energy retrofit: Life cycle cost benefit analyses using cash flow analysis and multiple benefits on project level. Energy Effic. 2019, 12, 261-279. [CrossRef]

69. Duran, Ö.; Lomas, K.J. Retrofitting Post-War Office Buildings: Interventions for Energy Efficiency, Improved Comfort, Productivity and Cost Reduction. J. Build. Eng. 2021, 42, 102746. [CrossRef]

70. Đukić, M.; Zidar, M. Sustainability of Investment Projects with Energy Efficiency and Non-Energy Efficiency Costs: Case Examples of Public Buildings. Sustainability 2021, 13, 5837. Available online: https:/ /www.mdpi.com/2071-1050/13/11/5837 (accessed on 25 September 2021). [CrossRef]

71. Local Law 97. New York City Charter; New York City Mayor's Office: New York, NY, USA, 2019.

72. SB-1477: Low-Emissions Buildings and Sources of Heat Energy; State of California: Sacramento, CA, USA, 2018.

73. Coffey, B.; Borgeson, S.; Selkowitz, S.; Apte, J.; Mathew, P.A.; Haves, P. Towards a Very Low-Energy Building Stock: Modeling the US Commercial Building Sector to Support Policy and Innovation Planning. Build. Res. Inf. 2009, 37, 610-624. [CrossRef] 